\title{
Two Dimensional Li Beam Imaging to Study the Magnetic Field Configuration Effects on Plasma Confinement in Spherical Tokamak CPD
}

\author{
R. BHATTACHARYAY, H. ZUSHI ${ }^{1)}$, T. MORISAKI ${ }^{2)}$, K. KIKUKAWA, S. WATANABE, \\ Y. INADA, M. HASEGAWA ${ }^{1)}$, H. TSUCHIYA ${ }^{3)}$, K.N. SATO $^{1)}$, K. NAKAMURA ${ }^{1)}$, \\ M. SAKAMOTO ${ }^{1)}$, H. IDEI ${ }^{1)}$, K. SASAKI, H. XU, F. WANG, S. KAWASAKI ${ }^{1)}$, \\ H. NAKASHIMA ${ }^{1)}$ and A. HIGASHIJIMA ${ }^{1)}$ \\ Interdisciplinary Graduate school of Engineering Science, Kyushu University, Kasuga, Fukuoka, 816-8580, Japan \\ ${ }^{1)}$ Research Institute of Applied Mechanics, Kyushu University, Kasuga, Fukuoka, 816-8580, Japan \\ ${ }^{2)}$ National Institute for Fusion Science, Toki 509-5292, Japan \\ ${ }^{3)}$ Graduate Universty for Advanced Studies, National Institute for Fusion Science, Toki 509-5292, Japan
}

(Received 4 December 2006 / Accepted 21 April 2007)

\begin{abstract}
Two dimensional lithium beam imaging technique has been applied in the spherical tokamak, CPD (Compact Plasma wall interaction experimental Device), to study the effects of various magnetic field configurations on RF plasma confinement topology. The performance of the lithium sheet beam is absolutely calibrated by a quartz crystal monitor. Experimental results show that plasma initiation takes place at the electron cyclotron resonance layer in a simple torus configuration and then it expands quickly to the low magnetic field side. Different magnetic field configurations critically affect the RF plasma confinement topology. A sharp lower boundary exists for the RF plasma in magnetic null configuration. Magnetic connection length plays the key role in defining plasma boundary and the critical value of connection length for plasma to exist in CPD is found to be $\sim 5-6$ meter for a given pressure condition.
\end{abstract}

(c) 2007 The Japan Society of Plasma Science and Nuclear Fusion Research

Keywords: lithium beam, spherical tokamak, plasma confinement, plasma boundary, magnetic field configurations, connection length, decay index

DOI: $10.1585 /$ pfr.2.S1103

\section{Introduction}

Nuclear fusion is considered to be a promising long term candidate for alternate energy source. To achieve fusion energy on earth steady state operation of high temperature and high density plasma is required. Tokamak is one of the prospective devices to confine high temperature and high density plasma. Recently, spherical tokamak (ST) concept is also getting considerable importance in the fusion community because it has the capability of sustaining high beta plasma in a compact shape of low aspect ratio and the cost of the reactor is also cheap [1]. The major engineering difficulty associated with the ST reactor concept is to accommodate the central solenoid for plasma start-up, since STs have only a limited space at the central column of the device to ensure its low aspect ratio $(<2)$ property. It is, therefore, strongly desired to develop a non-inductive current start-up scheme to initiate and ramp up the plasma current to a level which would then be sufficient for the auxiliary heating as well as current drive schemes to take over. Electron cyclotron heating is one of the potentially attractive candidates for this purpose, since plasma initiation and current start-up might be realized simultaneously.

author'se-mail: raju@triam.kyushu-u.ac.jp
It has also been realized for tokamak configuration that RF assisted inductive start-up results in faster and more reliable breakdown with a much higher tolerance to field null quality [2]. In fact, for ITER start-up with a multi-pole null magnetic field configuration, addition of an extra 3 MW RF power is planned along with Ohmic power to facilitate reliable breakdown over a wide range of error field and pressure conditions [3]. The possibilities of plasma current generation in RF plasma have been demonstrated in CDX-U [4] LATE [5] and TST-2@K [6] under a weak magnetic mirror configuration. Such current formation as well as ramp up under a steady $B_{z}$ field is considered to be responsible for the change of magnetic field topology from an open field equilibrium to a closed field equilibrium. It is, therefore, important to study the plasma confinement topology under various magnetic field configurations and the changes in confinement topology associated with the initiation of plasma current. Recently a two-dimensional density diagnostic system with a thermal sheet Li beam has been installed in the spherical tokamak CPD to obtain the density contour in the lower half of CPD plasma and to investigate the current start up physics associated with the change in magnetic field configurations [7]. In this paper, 
study of plasma confinement topology in CPD under various magnetic field configurations using the sheet lithium beam is being reported.

\section{Experimental Set Up}

\subsection{CPD and arrangement of Li beam imag- ing system}

CPD is a compact ST device $\left(R_{0}=0.3 \mathrm{~m}, a=0.2 \mathrm{~m}\right)$ with central solenoid (CS), four toroidal field (TF) coils and three sets of poloidal field (PF) coils. Since there are four toroidal coils, and the return coils are located far from the plasma area, the ripple is tolerable $(\sim 8 \%$ at $R=0.5 \mathrm{~m})$. The maximum current for each TF coil is $90 \mathrm{kA}$, which can produce $B_{\mathrm{t}}=0.25 \mathrm{~T}$ at $R_{0}$. A set of $8.2 \mathrm{GHz}$ klystrons $(8 \times 25 \mathrm{~kW}, \mathrm{CW})$ is used for ECH. Linearly polarized microwaves are injected through the rectangular horn antenna below the equatorial plane with an injection angle of $\sim 15^{\circ}$ from the normal direction to the toroidal field lines. In these experiments $2-100 \mathrm{~kW}$ of RF power is used to initiate and sustain the plasma. The two dimensional density imaging system ( $300 \mathrm{~mm} \times 500 \mathrm{~mm}$ ), attached to CPD, is composed of a sheet Li beam and a CCD camera (Pixelfly qe) equipped with an optical filter $(670.8 \pm 5 \mathrm{~nm})$ for monitoring LiI intensity. The Li beam injector is located at the bottom of the chamber and the beam is injected at an oblique angle of 18.18 degree at $\mathrm{R} \sim 0.29 \mathrm{~m}$ with respect to the machine axis, as shown in Fig. 1. The oven is heated up to a temperature of around $500{ }^{\circ} \mathrm{C}-520^{\circ} \mathrm{C}$ and the lithium beam is extracted through an array of eleven collimated holes, each of diameter $\sim 3.0 \mathrm{~mm}$ and is shaped through a rectangular slit $\left(35 \times 6 \mathrm{~mm}^{2}\right)$, whose longer side is aligned with the major radius of CPD. The sheet thermal beam is calibrated using a quartz crystal deposition monitor (QCM)

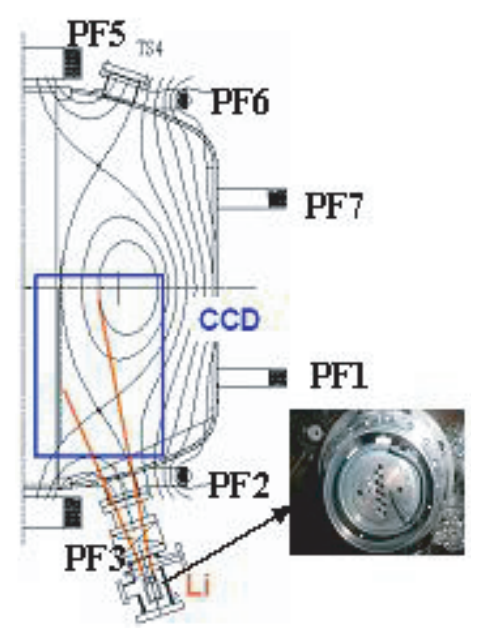

Fig. 1 Poloidal cross sectional view of CPD showing different $\mathrm{PF}$ coil positions and typical magnetic surfaces. Li injector is attached at the bottom and CCD camera is used to view the lower half of mid plane. The collimator on the top of the oven is shown separately. from the decrease in frequency of the quartz oscillator from $6 \mathrm{MHz}$ to $4.5 \mathrm{MHz}$. Since the effective size of the quartz crystal is $\sim 8 \mathrm{~mm} \phi$, the deposition flux (or evaporated $\mathrm{Li}$ flux, $\Gamma_{\mathrm{Li}}$ ) is evaluated from the Li film thickness and operation duration. By scanning the crystal sensor by $\pm 20 \mathrm{~mm}$ across the rectangular beam at the fixed position, the characteristics of the sheet beam are evaluated. The uniformity of $\pm 10 \%$ of $\Gamma_{\mathrm{Li}}$ is achieved for $40 \mathrm{~mm}$ along the long side of the rectangular slit ( along the major radius) and the full width at half maximum is found to be $\sim 40 \mathrm{~mm}$ along the short side of the rectangular slit (along the toroidal direction) at a distance of $\sim 0.47 \mathrm{~m}$ from the slit. The details regarding the performance of the sheet lithium beam can be obtained in Ref. [7]. The CCD camera has a tangential view into the chamber. 2D image (boxed area) can be recorded for $1-2 \mathrm{~ms}$ typically with $1392 \times 1024$ pixels with a size of $6.7 \mu \mathrm{m} \times 6.7 \mu \mathrm{m}$. The CCD readout time is $\sim 40.3 \mathrm{~ms}$ by pixel binning $(696 \times 512)$. A lens (PENTAX B1214D-2) whose focal length is $12.5 \mathrm{~mm}$ and F-number is 1.4 is attached in front of the CCD. The spatial resolution is $\sim 1 \mathrm{~mm}$. When an incident angle of the light deviates from the normal direction to filter surface, the transmission efficiency of the optical filter is reduced and the peak of the filtered wavelength is down shifted. The edge region of the boxed area (see Fig. 1) is hence interfered by $H_{\alpha}$ emission. Assuming plasma reproducibility with and without $\mathrm{Li}$ injection the CCD image of LiI intensity is obtained.

\section{$2.2 B_{z}$ profiles of various coils in CPD}

Fig. 2 shows the radial profile of vertical magnetic field $\left(B_{z}\right)$ at the mid plane $(Z=0)$ of CPD due to various magnetic field coils. The coil current is set to $1.0 \mathrm{kA}$ for each coil. Typical operational current values for each coil are, CS : 5 to $25 \mathrm{kA}$; PF2 and PF6 (denoted as PF26): -6.0 to $+6.0 \mathrm{kA}$; PF1 and PF7 (PF17): 0 to $3.0 \mathrm{kA}$. PF3 and PF5 (PF35) coils are not powered during this experimental campaign. It should be noted that $B_{z}$ produced by PF17 coil has a small negative decay index $\left(n^{*}\right)$, defined as,

$$
n^{*}=-R / B_{z} \times\left(\partial B_{z} / \partial R\right)
$$

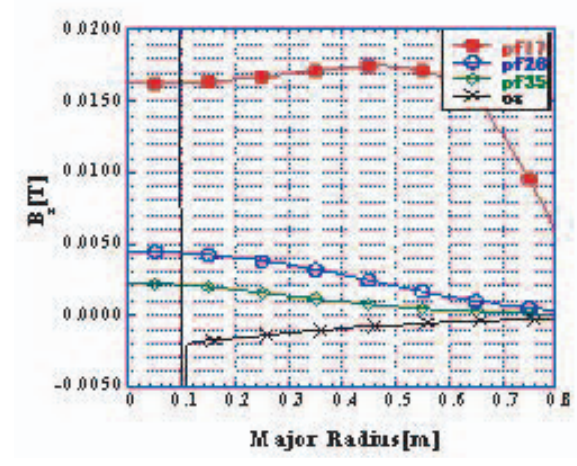

Fig. $2 B_{z}$ profile at the mid plane of CPD for various magnetic field coils. All coil currents are set to $1.0 \mathrm{kA}$. 
where as other PF coils along with CS coil produces $B_{z}$ with a positive decay index. Null magnetic field configuration is obtained by combining CS and PF26 coil currents. Moreover, the $B_{z}$ configuration of each of these coils is significantly different from the other. Hence plasma confinement topology could be studied under various types of magnetic field configuration.

\section{Experimental Results}

\subsection{Plasma formation in simple torus}

Linearly polarized microwaves are injected in both Xmode and O-mode from the outboard side at oblique angles, being tilted by $\sim 15^{\circ}$ with respect to the major radius. The toroidal coil current is raised linearly from $t=0 \mathrm{~s}$ up to $50-70 \mathrm{kA}$ in $t=0.1 \mathrm{~s}$ and is kept constant until $t=0.2-0.4 \mathrm{~s}$. For a given pressure condition an initial plasma is found to be instantly produced with the RF power of $\sim 2 \mathrm{~kW}$ at the position of the fundamental EC resonance $\left(B_{\mathrm{res}}=0.293 \mathrm{~T}\right.$ at $\left.R_{\text {res }}\right)$. Although at this break down phase the plasma stretches vertically as it is found by a fast camera, the vertically stretched LiI intensity contour is limited by the vertical width of the beam, as shown in Fig. 3. The expansion of this plasma column towards the low field side is not significant if the RF power is kept low, but with increasing RF power plasma quickly expands towards the low field side and over-dense plasma formation is observed. In fig.3 the maximum LiI light intensity at $R \sim 0.17 \mathrm{~m}$ indicates the position of fundamental resonance layer $R_{\text {res }}$ at $I_{\mathrm{TF}}=$ $60 \mathrm{kA}$ which agrees fairly well with the calculated value of $0.164 \mathrm{~m}$. It should be noted that the lower boundary is defined by the beam itself whereas the upper boundary is ob-

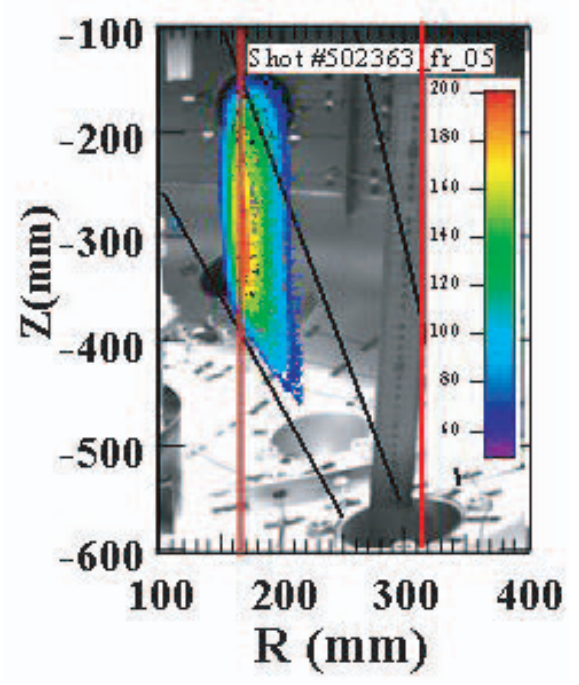

Fig. 3 2D image of LiI intensity contour for low power RF plasma in simple torus configuration. Three solid inclined lines indicate the coverage area of the sheet lithium beam. Vertical lines indicate the positions of fundamental and $2^{\text {nd }}$ harmonic resonance. Attached color scale is for LiI intensity in arbitrary unit (a.u.). served due to the reduction in the transmission efficiency of the filter. Observed boundary in the radially outboard side confirms that there is no plasma beyond $R \sim 0.23 \mathrm{~m}$. Observed boundary in the radially inboard side indicates either complete attenuation of beam or enhanced loss of plasma at the center stack cover whose position is at $R \sim$ $0.12 \mathrm{~m}$.

\subsection{Plasma confinement topology in mag- netic null configuration}

2D image of LiI intensity contour for magnetic null configuration is shown in Fig. 4 along with the poloidal flux contour. Null configuration is produced by the combination of CS and PF26 coil currents. It is clear from the figure that a clear lower boundary exists for the LiI image. The observed radially outboard boundary is due to the Li beam coverage limit and upper boundary indicates the complete attenuation of the beam. The electron density is found to be significantly higher than the cut-off density. It is to be noted here that even by varying the RF power within 10-50 kW and individual coil current (but keeping the same magnetic configuration) no significant change is observed in the observed lower boundary. Only image is found to get broaden in the upper direction by lowering $\mathrm{RF}$ power which indicates more penetration of the $\mathrm{Li}$ beam due to lower electron density. The observed lower boundary of LiI image is found to correlate well with the calculated poloidal flux contour. Below the image boundary the poloidal flux lines are bent downward and slightly above the boundary position it starts bending upward. Observed plasma boundary is thus considered to be due to the effects of magnetic field configuration.

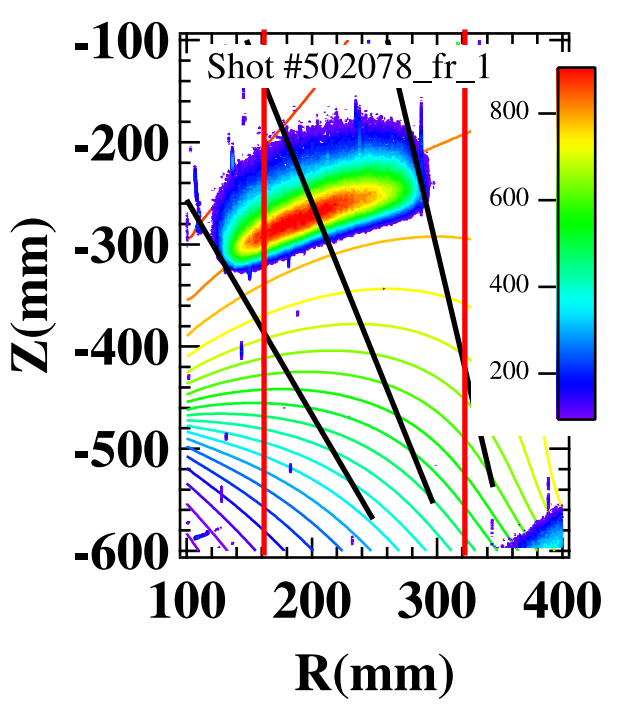

Fig. 4 2D image of LiI intensity contour is superimposed on the poloidal flux contour of magnetic null configuration. The attached color scale is for LiI intensity (a.u.). Vertical lines indicate the positions of fundamental and $2^{\text {nd }}$ harmonic resonance. 

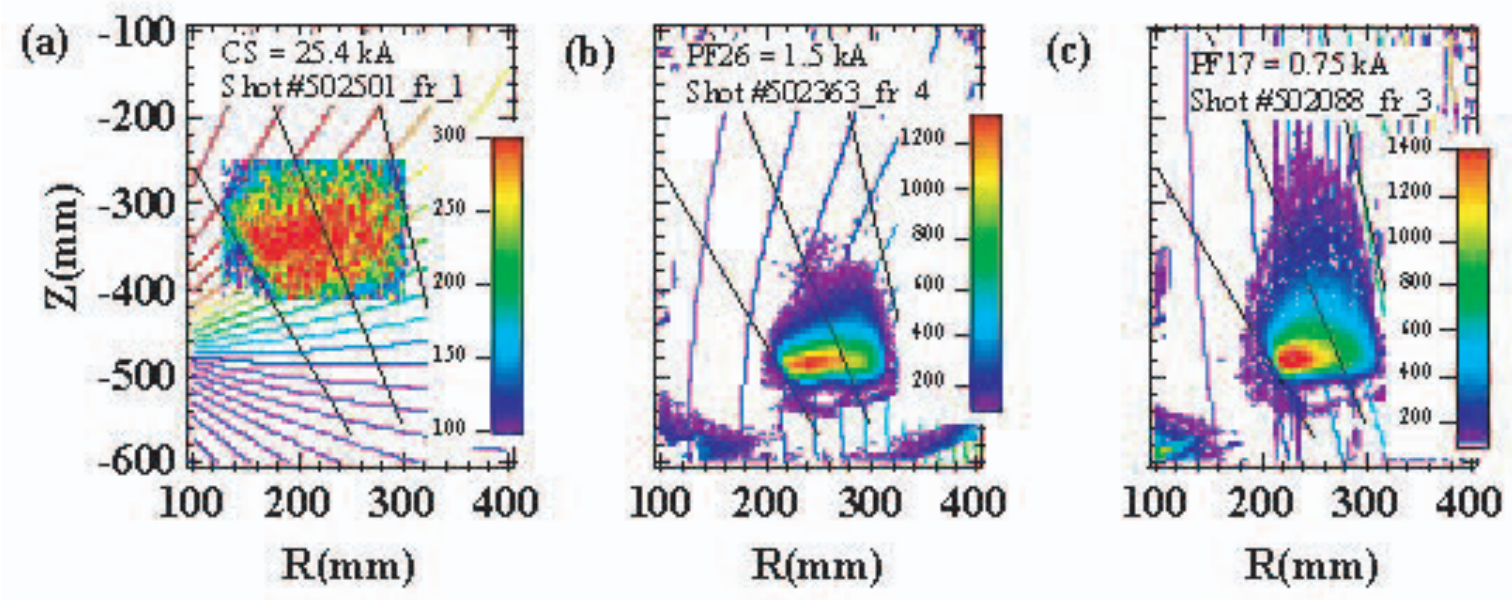

Fig. 5 2D image of LiI intensity contour is superimposed on the poloidal flux contour for the magnetic configuration of (a) CS coil $(I=$ $25.4 \mathrm{kA})$, (b) PF26 coil $(I=1.5 \mathrm{kA})$ and (c) PF17 coil $(I=0.75 \mathrm{kA})$. Attached color scales are for LiI intensity (a.u.).

\subsection{Plasma confinement topology in weak mirror configurations with $n^{*}$ of $\mathbf{- 0 . 0 6}$ $\sim+0.6$}

It has been mentioned earlier that the mirror configuration for individual PF coil and CS coil is significantly different from each other. Figures 5 (a)-(c) show the poloidal flux contour for CS $\left(n^{*}=0.6 @ R=0.25 \mathrm{~m}\right)$, PF26 $\left(n^{*}=0.4 @ R=0.25 \mathrm{~m}\right)$ and PF17 $\left(n^{*}=-0.06 @\right.$ $R=0.25 \mathrm{~m}$ ) coils. The coil currents are specified in the figure. Images of LiI contour are also superimposed on the respective poloidal flux contour. It is observed that a clear lower boundary exists again for the mirror configuration of CS but for PF26 and PF17 mirror configurations no such lower boundary is observed. The observed boundary for $\mathrm{CS}$ is different from that of null configuration and it remains unaffected with the variation of RF power as well as coil current magnitude. It should be noted here that though the decay index for PF17 coil is negative (negative mirror) as compared to PF26 coil which has a positive decay index, no change is observed in the lower boundary of the contour images for those two cases. The observed difference in the image pattern in the upward direction is considered to be due to the difference in RF power level for the two cases. For PF17 coil case RF power is $45 \mathrm{~kW}$, but for PF26 coil $\mathrm{RF}$ power is $100 \mathrm{~kW}$ and hence it is considered that $\mathrm{Li}$ is strongly attenuated within a very small distance because of high density plasma formation. By changing the coil current magnitude and $\mathrm{RF}$ power some changes are observed in the image patterns, but no change has been observed in the lower plasma boundary.

\section{Discussions}

From all the experimental results shown in the previous section it is considered that the observed lower plasma boundary in each case, except simple torus configuration, is a consequence of magnetic field configuration itself. It
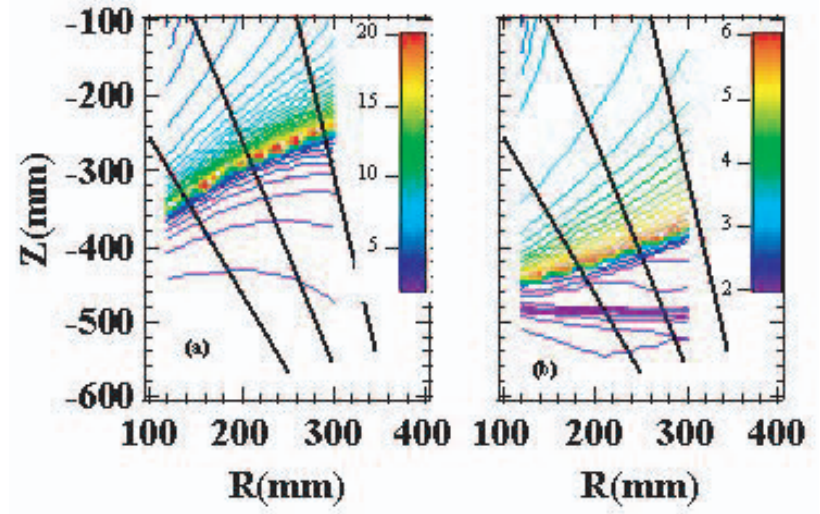

Fig. 6 Contour of connection length (wall to wall) for (a) magnetic null configuration; (b) CS magnetic configuration. Attached color scale indicates connection length in meter.

is well known that the main considerations for achieving a successful plasma breakdown are: (i) Townsend avalanche condition; and (ii) magnetic connection length. But once the breakdown occurs, connection length then should play an important role to define the plasma boundary. It is, therefore, worth to calculate the connection length for the above mentioned magnetic configurations and compare them with the experimental observations. Fig. 6 shows the contour of connection length for null magnetic configuration and for CS magnetic configuration. It is found that the connection length contours nicely reproduce the observed plasma boundaries (see Fig. 4 and 5 (a)) and the critical connection length for the existence of plasma is found to be $\sim 5-6 \mathrm{~m}$. The electron density, however, is found to be relatively low $\left(\sim 10^{16} \mathrm{~m}^{-3}\right)$ just near the plasma boundary. For PF17 and PF26 magnetic configurations, connection length is found to be much higher ( $\geq 20 \mathrm{~m}$ ) even near the port location of $\mathrm{Li}$ beam injection and hence no bound- 
ary is found. These experimental results suggest that for small size tokamak RF assisted plasma breakdown as well as sustainment may be possible even with a short effective connection length. Though RF driven plasma current (0.5$1.0 \mathrm{kA}$ ) has been found to exist in presence of $B_{z}$ of $\sim 50 \mathrm{G}$, associated changes in the vertically stretched plasma are still not clearly observed. Since the viewing area is limited up to $-100 \mathrm{~mm}$ below the mid plane, co-existence of circular plasma along with vertically stretched plasma may be expected. Dedicated experiments with careful adjustment of PF coil currents will be planned in near future to study the changes in plasma boundary with the onset of plasma current.

\section{Conclusion}

Plasma confinement topology has been studied in CPD spherical tokamak using two dimensional thermal sheet lithium beam imaging technique. Plasma confinement topology is found to change significantly with the change in magnetic configuration. Magnetic connection length plays an important role in defining the plasma boundary and for CPD the critical connection length for plasma to exist is found to be $\sim 5-6 \mathrm{~m}$.

\section{Acknowledgement}

This work has been partially performed under the framework of the bi-directional collaboration organized by NIFS. This work is partially supported by a Grant-in-Aid for Scientific Research from Ministry of Education, Science and Culture of Japan.

[1] H.R. Wilson et al., Nucl. Fusion 44, 917 (2004).

[2] B. Lloyd et al., Nucl. Fusion 31, 2031 (1991).

[3] ITER Phys. Basis, Nucl. Fusion 39, 2605 (1999).

[4] C.B. Forest et al., Phys. Plasmas 1, 1568 (1994).

[5] T. Maekawa et al., Nucl. Fusion 45,1439 (2005).

[6] A. Ejiri et al., Nucl. Fusion 46, 709 (2006).

[7] H. Zushi et al., PSI17, P3-58, China, May 22-26, 2006 (In press, J. Nucl. Mater (2007) doi:10.1016/j.jnucmat.2007. $01.259)$ 